\title{
Energy Efficient of IDS Using Fuzzy Logic for Lifetime Improvement in Wireless Sensor Network
}

\section{S. Venkatesan, M. Ramakrishnan}

\begin{abstract}
The Wireless Sensor Network (W.S.N.) comprises little batteries fueled sensor gadgets with restricted energy assets. The Sensor hubs used to monitor the physical screen or conditionsbased on normal, theinformation must be private organization to primary area. The Most significant obstacles in a sensing the remote in the particular network which used to make an efficient energy framework. Clustering is the one of the major process in the sensor network based on wireless which used to drag out the life time of an organization lifetime which in turn reduce the energy utilization of the network. It includes gathering hubs into groups and choosing bunch heads $(\mathrm{CH})$ for all the groups. $\mathrm{CH}$ gather information from separate group hubs and forward the collected data to the fundamental corner. This paper proposes novel fluffy various dynamic methodology measures: "Energy Efficient Optimal Cluster Head Selection utilizing Fuzzy Logic (EEOCH-FL)" for Wireless Sensor Network. Fluffy different boundary dynamic methodology is used to choose C.H.S utilizing three standards: leftover energy, fixation, the right ways from the principle hubs, and base station. The life cycle of Clustering hub and Clustering Head are grouped, clustering hub which transmitted all data to the Cluster Header Leader (CHL). The bunch head pioneers sent collected information to the Base Station (B.S.) from that point forward. The determination of bunch heads, group head pioneers is controlled and monitored by utilizing a fluffy rationale. The information transmission measure is per-shaped by the briefest energy way chosen to apply Dijkstra Algorithm. The reenactment results show that this methodology is more potent in boosting the availability inside each bunch. Furthermore, the reproduction aftereffects of this examination are contrasted and different conventions LEACH and CEELRP to assess the proposed steering convention's presence. The assessment reasons that convention of steering of this proposed work proved to be an effective in utilization of an energy.
\end{abstract}

Keywords: Intrusion Detection System, Fuzzy Logic, Lifetime of Network, Clustering, Routing protocol

\section{INTRODUCTION}

W ireless Sensor Networks (W.S.N.) is a gathering of spatially scattered devoted sensors to screen/record a climate's states of being and to sort out gathered information at a focal area [1]. Grouping is utilized for correspondence among hubs and B.S., the energy proficient contrasted with

Manuscript received on January 30, 2021.

Revised Manuscript received on February 04, 2021.

Manuscript published on February 28, 2021.

* Correspondence Author

S. Venkatesan*, Computer Application, Madurai Kamaraj University, Madurai, India, venkatesan708@gmail.com

Dr. M. Ramakrishnan, School of IT, Madurai Kamaraj University, Madurai, India, ramkrishod@gmail.com.

(c) The Authors. Published by Blue Eyes Intelligence Engineering and Sciences Publication (BEIESP). This is an open access article under the CC BY-NC-ND license (http://creativecommons.org/licenses/by-nc-nd/4.0/)

"single/multi-jump directing". In bunching, a sensor hub in a group is chosen "Cluster Head (C.H.)" and transfers information from a sensor collector [2, 3, 4]. Hardly any C.H. hubs are intensely stacked, in bunching when energy consumption occurs. CH performs conglomeration work on information got and sends it to B.S. where it is required. The filter is a mainstream steering convention utilizing bunch based directing to diminish energy utilization [1].LEACH partitions correspondence into adjusts with around

Including a setup stage and a consistent state stage[5,6,7].

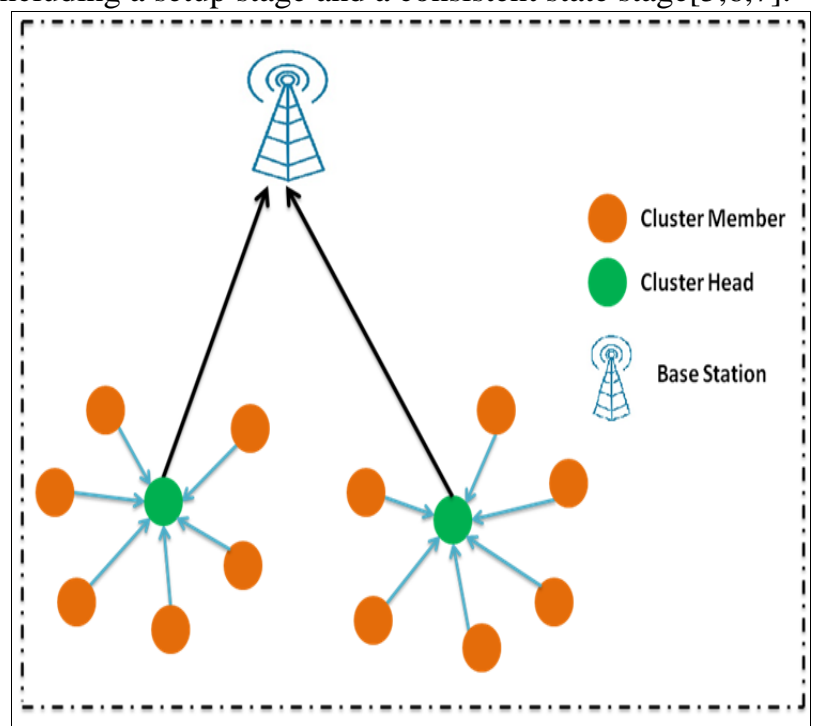

Fig.1. WSN Cluster Based - General System Model

An Energy Proficient Convention based on the Steering provided by Clustering. In a bunch, a hub is chosen as C.H. In contrast, others are part hubs who, in their groups, sense surrounding conditions in the climate and communicate estimated information to relating C.H.s [8]. C.H.s gather information from part hubs, total them, lastly moved to either multiple jump (CH) or single Jump (Straight forwardly) to B.S. Grouping which used to communication between the different hubs, and which used to transmit the data to the longest distance to BS[9]. Each Hub with Sensor used to gathering the related solitary group and interfaces to particular CH [10,11]. Henceforth, this implies proper C.H. must be chosen to improve "the C.H.'s utilization"; If not really, it might cause the passing of C.H. due to extra load for information assortment and sending. A large number of the directing calculations that depend on bunch strategy initially select C.H. aimlessly or by likelihood and structure the group. This examination essentially centers around numerous C.H.s and

Published By:

Blue Eyes Intelligence Engineering and Sciences Publication 
C.H.L.s, which are utilized in the enormous zone organization to communicate information to the base station and its information transmission cycle. These C.H.s and C.H.L.s are chosen by using a fluffy rationale. This examination endeavors to limit the absolute energy utilization and drag out this enormous territory organization's organization lifetime. This Paper provides a necessary information about contrasting the outcomes with deference with fluffy principle choice using LEACH and Ant Colony improvement calculations. Segment 2 records the writing of past work; Section 3 delineates the proposed strategy, Section 4 clarifies the outcome's examination, and Section 5 incorporates the ends.

\section{RELATED WORKS}

This work aims to investigate an Energy-Efficient Cluster head determination plot dependent on Fuzzy rationale for W.S.N. The current bunching calculations contrast the standards for the determination of the C.H.s. As per the flow research discoveries, the bunching based directing convention sees that each C.H. inside a group conveys the duty of bringing the message to the base station. This segment examines the vast majority of the notable grouped based steering calculations. As the proposed convention focuses on fluffy rationale idea, hardly any past works about the determination strategy for the C.H.s can be summed up as follows. "LEACH(Low Energy Adaptive Clustering Hierarchy)", the most eldest style "self-putting" together versatile grouping directing calculations for W.S.N.s in the olden days, which midpoints heap energy of entire organization to "the sensor hub"leads to customary C.H. political decision [12]. It can assist in improving the versatility and power of dynamic organizations somewhat. In the C.H. political race stage, every hub will create a varying number somewhere in the range of zero's and ones. This Number Varied not exactly "the edge T(n)", its will be considers as "the $\mathrm{CH}$ Hub". If this varying number is not exactly the edge T(n), it will be chosen as the C.H. hub. The meaning of $T(n)$ is impacted by the boundaries, for example, likelihood to be the C.H., current round, and the level of permitted C.H.s in the entire organization, which is appeared as Equation (1). $T(n)= \begin{cases}\frac{p}{1-p\left[\operatorname{rmod}\left(\frac{1}{p}\right)\right]}, & n \in G \\ 0 \quad, & \text { other }\end{cases}$

(1)

Assumed that $\mathrm{p}$ be the political race of $\mathrm{CH}$ during the level, $\mathrm{r}$ termed as the current $\mathrm{CH}$. Policatical

where $\mathrm{p}$ shows the level of hubs to be C.H. during the political race, $r$ is the current C.H. political race round, and G is the arrangement of hubs which neglect to be chosen as C.H. in the ongoing 1/p adjusts. Here the likelihood of every hub to be C.H. is the equivalent. From that point onward, C.H.s apportion schedule opens to the part hubs in the bunch. The information transmission inside the group will be performed with "TDMA (Time Division Multiple Access) "ways. Even though "LEACH" excessively unique thoroughly the correspondence utilization of genuine transmission of climate, the irregularity of C.H. political decision causes numerous insufficiencies, LEACH gives a decent hypothetical premise and configuration model for the ensuing grouping calculations. In [2], "LEACH-C "provided a unified way to deal with choosing the C.H. B.S. utilizes the harmonious way to deal with the position data and individual hub energy. B.S. shapes better bunches by circulating C.H. hubs all through the organization. The principle disadvantage of "LEACH-C" is talked about in [20]. "PEGASIS" altered adaptation of "LEACH " talked about in [3], the hubs speak with their nearest neighbors and structure a chain to convey the message to B.S. Another begins just a series corners speaking with "the BS" close. In [4]-[10], A Cluster-based energy productive area steering convention (CEELRP)[10] is an area-based directing convention. It applies the voracious calculation to chain the bunch heads. Many grouping conventions have been talked about. "HEED (Hybrid and Energy-Efficient Distributed grouping approach)" sets up an essential bunching boundary and an auxiliary bunching boundary during C.H. political decision as a proportion of the expense of intra-group correspondence [13]. The actual edge is subject to the hubs' remaining energy, and the hubs winter section higher likelihood energy of being a $\mathrm{CH}$. The optional border reacts the hub's vicinity or the hub's thickness, which is utilized as a helper pointer to calculate correspondence cost inside the bunch. This plan fabricates bunches in a dispersed manner, broadens the organizations' lifetime by limiting the intra-group correspondence energy utilization, and saves a proper conveyance for the C.H. hubs. There are substantially more messages that should have been communicated in group development, which brings about more extra framework energy utilization. "EADEEG (Energy-Aware Data Energy-Efficient Gathering convention for remote sensor organizations)"energy effective proposed an information combined with convention dependent on structured group, additionally sort of appropriated bunching calculation [14]. Considered the plan of energy hub and hub nearest energies which simultaneously used in $\mathrm{CH}$ political race. It can diminish the energy utilization and drag out the lifetime of the organizations somewhat. In any case, the plan overlooks the part of hubs degree, which may effectively prompt separated point issues of the organizations. "DSBCA (Distributed Self-association in burden Balanced Clustering Algorithm for remote sensor organizations) "characterizes group range limit to accomplish inconsistent bunching [15]. In $\mathrm{CH}$ political decision stage, new C.H. hubs are chosen to sort out the loads controlled by lingering energy and hub availability of every part hub, assessed by the previous C.H.s. Even though it could acquire the ideal C.H.s, the organization's energy utilization expanded. "EEREG (Energy Efficient Routing convention dependent on Evolutionary Game hypothesis)" sorts the suitable group size, reasonable C.H.s through game hypothesis [16]. In any case, it is a sort of brought together calculation with the consciousness of the organizations' worldwide geography. Furthermore, Sensor hub requires prerequisites equipment in higher order of the sensor hub. "CHEF (Cluster Head Election component utilizing Fuzzy rationale in remote sensor organizations)" is a sort of grouping calculation that brings fluffy rationale into remote sensor organizations to upgrade the energy utilization of the framework [17].

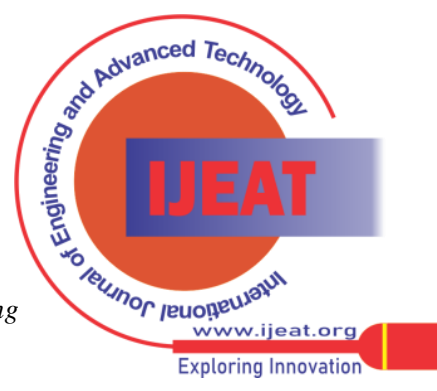


Cook picks hubs remaining energy and Distance among hubs and the base station as the info esteems to figure the likelihood of every hub to be C.H. Even though the calculation offers a decent choice plan for remote sensor networks in conveyed C.H. political decision, it disregards the impact of hubs degree on the energy use productivity for grouped organizations. "DFLC (Distributed Fuzzy Logic-based Clustering calculation)" is additionally a conveyed bunching calculation utilizing fluffy methodology for W.S.N.s [18]. It diminishes the number of pointless information bundles the hand-off hubs get an advance message while transferring from leaf hub to the root hub. Also, it takes out the hubs' statement, which has a lower likelihood to be another root. It adds a channel component before C.H.s political race to improve the applicant hubs' nature at the end of the day. This calculation picks hub lingering energy, the base station distance, hubs thickness thought contribution factor to fluffy methodology. A yield boundary "likelihood" is a fluffy etymological variable with five phases of fluffy division. A yield which changed over to a new incentive the "Center of Area (C.O.A.)" strategy. Nonetheless, it overlooks the hotspots issue in multi-bounce correspondence. It might load which causes awkwardness and lead to a prior death of hand-off hubs, which influences the entire organization frameworks' public exhibition. Also, considering the Distance among hubs and B.S. isn't exactly appropriate in the unique organization because the area of hubs is questionable in influential organizations, Thiswill cause a ton energy of utilization, where these hubs need to speak with B.S. the distance of the current progressively in each rounds. "LAUCF (Low-energy Adaptive Unequal Clustering convention utilizing Fuzzy c-implies)" is a low energy versatile inconsistent bunching calculation using fluffy c-intends to choose reasonable C.H. hubs to uniform energy utilization [19]. In [20], the creators additionally proposed a fluffy c-implies grouping calculation to spare energy utilization. The group head is chosen dependent on the sensor's area inside each bunch, its place as for combination focus (F.C.), its sign-to-commotion proportion (SNR), and its excess energy. In [21], the creators utilized fluffy c-intends to group organizations to lessen the briefest way blunder between far hubs and improve the last restriction exactness for sporadic intellectual radio organizations limitation. A Result based on fluff investigation of c-mean more sensible, hubs require considerably more additional energy utilization during each C.H. political race. "DUCF (Distributed Unequal Clustering utilizing Fuzzy rationale)" is a conveyed bunching calculation dependent on the fluffy rationale for inconsistent grouping organizations, which picks hub lingering energy, hub degree, and distance among hubs, base station information, picks likelihood chosen C.H. and group size yields [22]. A plan take a variables hubs and size of the group into thought and upgrades its general presentation. Like the deficiencies in DFLC, getting the Distance among hubs and B.S. continuously likewise prompts a ton of energy utilization in a unique organization, which affects sensor hubs life times.

Considered the insufficiency calculations referenced above, proposed an "Energy Efficient Distributed Clustering calculation utilizing Fuzzy methodology" a non-uniform sensor network dissemination referred as "EEDCF". This measure completely conveyed bunching calculation that every hub utilizes a TSK fluffy deduction framework to dissect whether it fits the C.H. contrasted and neighboring hubs. Hubs just need to speak with the neighbor hubs to keep up the nearby geography. We characterize the information boundaries as hub remaining energy, hub degree, and neighbor hubs' lingering energies. The fluffy rationale is proper for settling on endless choices with multi-causal components without complete climate for the organization sensor hub.This neighbor hubs' present excess an extra information esteem which can enhance the heap equilibrium of transfer hubs, maintain a strategic distance from the hotspots issue brought about by multi-bounce correspondence and it will be more useful to the advancement of energy utilization ,expand the organization lifetime framework.

\section{PROPOSED ROUTING PROTOCOL - NETWORK MODEL}

The network model of this study is shown in Fig 2. This routing protocol provides a balance in energy consumption and prolongs the network lifetime.

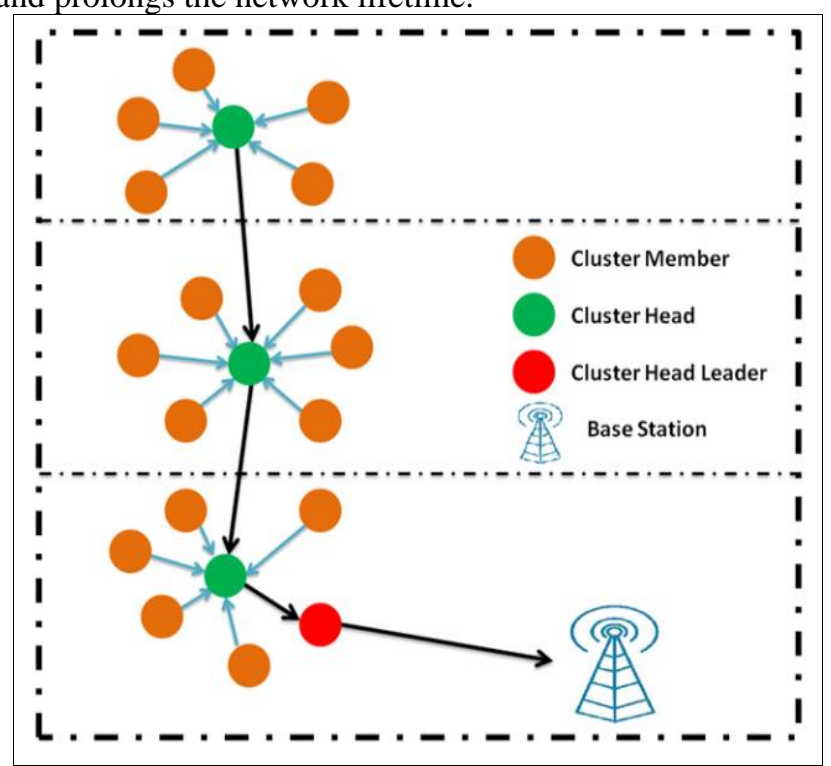

Fig 2. Proposed Network Model

The proposed routing protocols put together groups so all the hubs can be remembered for these bunches. It picks C.H.s for each club utilizing Fuzzy rationale, which depends on the most elevated energy resume and the B.S.'s least good ways. The Dijkstra calculation applied to locate the energy briefest way every hub. From that point onward, it courses the bunch individuals and C.H.s as per most straightforward energy ways. At long last, group individuals; send information parcels to the C.H.s. In this convention, C.H.L.s are picked by the B.S. utilizing fluffy rationale dependent on the most impressive energy resume and least good ways from B.S. of each C.H. C.H.L. can send information straightforwardly, contingent upon the most limited energy way. We reproduce this organization to break down Packet conveyance proportion, End End Delay, Throughput, Energy utilization, Number of Alive Nodes of this examination. Reproduction results show that convention is effective in dragging out organization duration and utilization of energy which contrasted with the LEACH and CEELRP.

\subsection{System Assumption}

Published By:

Blue Eyes Intelligence Engineering and Sciences Publication (C) Copyright: All rights reserved.

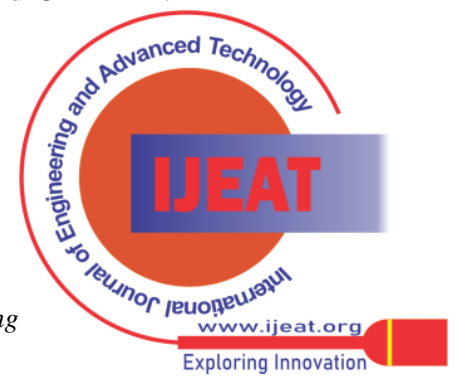


This Research Work will describe the sensor hubs that are viewed consistently to screen the climate ceaselessly.

1. Every sensor hubs viewed as static in the Base Stations.

2. Organization based on homogeneous which views as the end goal where all the hub based on sensor starting energy equivalent.

3. Determine the distance between the sensor hub and base station dependent on "signal strength maker (RSSI)"

4. A backup C.H. (C.H.L.) is chosen in the last degree of the chain (closer to the B.S.) for conveying the message to B.S. Final $\mathrm{CH}$ will be happens as drop out during the energy occurrence.

A Model based on proposed approach C.H. chosen dependent F.L. model which survey t F.L. can deal with more significant level vulnerabilities present in proper mind-boggling conditions [23]. The Model proposed is portrayed in Fig 3.

\subsection{Proposed Algorithm}

1) Consider Sensor Nodes as N, which act as randomly distributed in the form of M.X.M. region, let $k$ be assumed as a cluster.

2) Select $\mathrm{CH}$ from each level focused on the Model related to F.L.

3) Node[i].statement equal to initialize the stage for each node[i]

4) In the sensor, collect and send the appropriate data to the Current CH Node

5) The Parameters such as "Residual Energy(RE)", "Concentration (CO)", "Distance to BS(DS)"

6) Assume that residual energy of a node[i] equal to Node[i].RE

Assume that Concentration of each node[i] equal to Node[i].CO

Assume that distance of node[i] equal to Node[i].BS

7) "Fuzzy Inference System (FIS)" should be analysis Determine the probability of FIS such that three parameters should be passed( node[i].RE,node[i].CO,node[i].BS)

8) By Electing the node of $\mathrm{CH}$, Compare the result with FIS such

if node.[i] probability is greater than the node[j].probability, then $\mathrm{CH}$ will assign to Node.[i].statement.

9) A Message of C.H. should be advertised

10) otherwise

11) C.H. Messages while receiving, the nearest node must be selected.

12) NODE_JOIN to the nearest C.H. will be send

13) Stop

14) If an exception occurs in C.H.

15) If the node[C.H.] probability will be less than I node[j] probability, which changes the Node[C.H.].statement equal to a fault.

16) Select a New $\mathrm{CH}$, then

17) Otherwise

18) Terminate

19) Stop

20) The Data should be transmitted from the upper lever still C.H. reaches its or other base stations.

21) A Sensor node with Energy higher elected by the CHL which close to the Base Station with Resume connectivity, in case if any failure occurs at the end $\mathrm{CH}$ ( BS consumable energy closer to $\mathrm{CH}$ )

22) end
23) From the Route of Data Transmission, Base Station which collects the data aggregated.

24) End

\section{SIMULATIONRESULTS}

This segment talks about the reenactment of this examination. The reproduction happens by utilizing Network Simulator 2. We utilize the Fuzzy Inference Engine to choose C.H.s and C.H.L.s. We additionally apply the Dijkstra calculation to chain the group individuals as per their most straightforward energy way. Recreation settings for EEOCHFL convention execution are demonstrated as follows. The recreation boundaries for the assessment of the energy effective bunch head determination appeared in table IV. By considering the fixed organization size of 1000x1000 $\mathrm{m} 2$ and 150 hubs are made. The underlying energy of each hub was set to 1 Joules. The information parcel size is 780 pieces, and the information transmission time is 10 msecs. For the "EEOCHFL" steering convention, the distinctive execution boundaries are thought of, and they are characterized as described.

1. Packet conveyance Ratio - The proportion of the measure of adequately got parcels to the aggregate sum of bundles sent by the sender during transmissions.

2. End to End Delay - The time being used by the information bundles to arrive at the objective hubs. As the average start to finish time diminishes in an organization, the presentation pointer esteem also increments a decent organization execution.

3. Energy Consumption - It is communicated as the all-out energy used by sensor hubs during the transmission period. The number of bunches and hubs, a base station area, and adjust time are necessary to legitimize hub energy.

4. The number of alive hubs - A MANET is a controlling framework, as hubs run with limited force batteries. Expanding the organization's lifetime depends on compelling controlling of hub energy. By creating the most extreme hubs to be alive, the lifetime of the organization can be expanded.

Table 1: Simulation Parameter of EEOCHFL Protocol

\begin{tabular}{|l|l|}
\hline Type of Parameter & Value \\
\hline Propagation & Two ray ground \\
\hline Field size- m x m & 1000 x 1000 \\
\hline Number of nodes & 150 \\
\hline Channel Type & Wireless Channel \\
\hline Antenna Type & Omni \\
\hline Link Type & L.L. \\
\hline $\begin{array}{l}\text { The initial energy of } \\
\text { MANET node- Joules }\end{array}$ & 1 \\
\hline Data packet size- & 780 \\
\hline Simulation time-ms & 50 \\
\hline Transmission time-ms & 10 to 50 \\
\hline Observation Parameters & $\begin{array}{l}\text { Packet Delivery Ratio, End to End Delay, } \\
\text { Alive Nodes, Energy Consumption, }\end{array}$ \\
\hline
\end{tabular}

\subsection{Simulation Results Of Eeochfl} Protocol

Published By:

Blue Eyes Intelligence Engineering and Sciences Publication

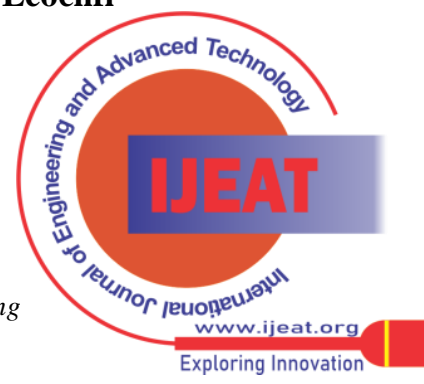


In this examination work, the creator endeavored to improve the organizations' exhibition by estimating a portion of the related and trustworthy qualities taken into thought. The exhibition factors are estimated by dissecting and reenacting the exploratory arrangement utilizing the EEOCH-FL convention. The creator organized the reproduction results that got utilizing this convention and related to LEACH and CEELRP convention's consequences. Here, the reenactment results are acquired for the presentation boundaries start to finish delay, parcel conveyance proportion, energy utilization, number of alive hubs from the got recreation results;

\section{a) Packet Delivery Ratio}

Fig 3. Shows correlation of the exhibition factor the parcel conveyance proportion against the number of hubs for the proposed EEOCHFL, LEACH, CEELRP conventions. The quantity of hubs differs from 10 to 150 . The parcel conveyance apportion of each plan is resolved, and the outcome shows that the proposed work conveyances more number of bundles to the based station because of the ideal choice of bunch head. This because of the determination of bunch head with most minimal part transport estimation of good ways from the base station to group head and the non-bunch head thickness with the assistance of fluffy Logic different rules dynamic methodology based bunching and age of promising hubs as the populace in each round, appointment of group head with most noteworthy wellness esteem with the Distance as the factor.

Alive Nodes: Figure 3 is shown of the number of alive hubs in LEACH, CEELRP, and EEOCHFL Protocol for directing convention. The acquired consequences of the number of alive hubs obtained from reproduction results utilizing NS2 are classified in Table 2.

Several Nodes Alive more than Several Rounds Another significant issue in W.S.N. is the number of hubs alive more than a few rounds. In this segment, Figure 11 presents the number of lifetimes of hubs, which implies the round quantities until the main hub passes on for our convention. Figure 11 shows that it is higher than LEACH and CEELRP. We also note that the lifetime diminishes at cycle 150 in LEACH and cycle 220 for CEELRP. On account of FEMCHRP, the abatement just beginnings after more than 410 rounds. That implies an average number of live sensor hubs in EEOCHFL is 17\% higher than LEACH, 10\% higher than CEELRP. Consequently, it has been demonstrated that EEOCHFL is better in drawing out organization lifetime contrasted with the LEACH and CEELRP.

Table 2: Measure of Number of Alive Nodes LEACH, CEELRP vs. EEOCHFL Protocol

\begin{tabular}{|c|c|c|c|}
\hline \multirow{2}{*}{$\begin{array}{c}\text { Number } \\
\text { of nodes }\end{array}$} & \multicolumn{3}{|c|}{ Number of Alive Nodes } \\
\cline { 2 - 4 } & LEACH & CEELRP & EEOCHFL \\
\hline 10 & 10 & 10 & 10 \\
\hline 25 & 18 & 21 & 23 \\
\hline 50 & 34 & 37 & 42 \\
\hline 75 & 47 & 53 & 60 \\
\hline 100 & 69 & 76 & 81 \\
\hline 150 & 108 & 114 & 129 \\
\hline
\end{tabular}

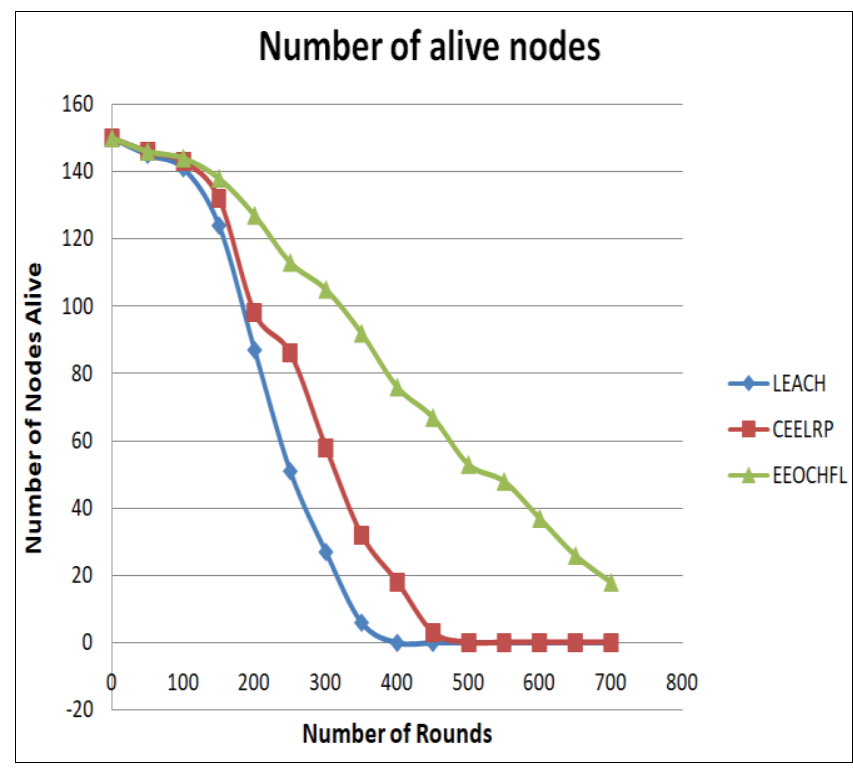

Figure 3: Number of Alive Nodes vs. Number of Nodes

\section{V.CONCLUSIONS}

The limitation of energy in sensor hubs challenges the plan and improvement of conventions for remote sensor organizations. Considering that energy effectiveness is one of the first and most explored issues in remote sensor organizations, energy proficient directing convention is introduced utilizing the fluffy Logic grouping calculation. From the writing review, it was discovered that LEACH convention indicated better execution and radiate highlights are acquired as far as execution factors like deferral, energy utilization, P.D.R. and so forth the significant inadequacy of LEACH convention is choosing C.H. This downside is wiped out by proposing an improved convention EEOCHFL by considering the number of hubs up to 150 . The restatements of this investigation are examined beneath. First, this organization devours less energy to communicate all out accumulated information to the Base Station than different conventions. Its energy utilization is a lot lower than $\mathrm{LEACH}$ and CEELRP. Second, the organization lifetime begins diminishing after more than 410 rounds, which is a lot higher than different conventions and implies that this convention is superior to different conventions as far as organization lifetime. From the acquired recreation results utilizing NS2 apparatus, the exhibition factors P.D.R., End to end delay, throughput, directing overhead, energy utilization, number of dead hubs, number of alive hubs shows better execution regarding energy utilization, life season of the organization, and area of the group with most short Distance. This proposed strategy improvement happened regarding P.D.R. by $13.62 \%$, start to finish delay by $16.37 \%$, energy utilization by $34 \%$, and the number of alive hubs by $10 \%$ contrasted with LEACH and CEELRP convention. The weaknesses of execution measures might be improved in element by fusing fluffy rationale and hereditary calculation idea during information transmission in W.S.N. With by and large outcomes, the proposed technique EEOCHFL convention diminishes generally speaking energy utilization and organization lifetime.
Blue Eyes Intelligence Engineering and Sciences Publication (C) Copyright: All rights reserved.

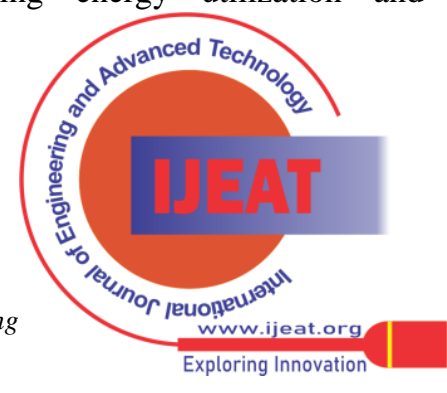


Energy Efficient of IDS using Fuzzy Logic for Lifetime Improvement in Wireless Sensor Network

\section{REFERENCE}

1. Abad, M.F.K. and Jamali, M.A.J. (2011) 'Modify LEACH algorithm for wireless sensor network,' IJCSI International Journal of Computer Science Issues, Vol. 8, No. 5.

2. Abraham, A., Jatoth, R.K. and Rajasekhar, A. (2012) 'Hybrid differential artificial bee colony algorithm,' Journal of Computational and Theoretical Nanoscience, Vol. 9, No. 2, pp.249-257.

3. Selvakumar, K., \&Selvi, M. S. (2014). Efficient Load, Balanced Routing Algorithm, Based On Genetic And Particle Swarm Optimization.

4. Manjusha, M. S., \&Kannammal, K. E. (2014). Efficient Cluster Head Selection Method For Wireless Sensor Network

5. Saini, M., \&Saini, R. K. (2013). The solution of Energy-Efficiency of sensor nodes in Wireless Sensor Networks. International Journal of Advanced Research in Computer Science and Software Engineering, 3(5), 353-357.

6. Tran Cong Hung and Ly Quoc Hung. Energy consumption improvement of traditional clustering method in wireless sensor network, International Journal of Computer Networks \& Communications (IJCNC) Vol.8, No.5, September 2016

7. Khalid, H., Abdullah, K.M., AhsanAwan, F., and Hussain, A. (2013) 'Cluster head election schemes for W.S.N. and MANET: a survey', World Applied Sciences Journal, Vol. 23, No. 5, pp.611-620.

8. Prasad, A. Y., and Balakrishna Rayanki. "A generic algorithmic protocol approaches to improve network lifetime and energy efficient using combined genetic algorithm with simulated annealing in MANET." International Journal of Intelligent Unmanned Systems (2019). Vol. 8 No. 1, pp. 23-42

9. Prasad, A. Y., and R. Balakrishna. "Implementation of the optimal solution for network lifetime and energy consumption metrics using improved energy-efficient LEACH protocol in MANET." Telkomnika Vol. 17 No.4 (2019): 1758-1766

10. Nurhayati, S. H.C., \& Lee, K.O (2011). A Cluster-based energy-efficient location routing protocol in wireless sensor networks. Proceedings international journal of computer and communication, 5(2)

11. Prasad, A. Y., and R. Balakrishna. "Optimized energy-efficient routing protocol for MANET using a fuzzy score based clustering algorithm" International Journal of Innovative Technology and Exploring Engineering. Volume-8 Issue-7, May 2019

12. Heinzelman, W.R.; Chandrakasan, A.; Balakrishnan, H. Energy-efficient Communication Protocols for

13. Wireless Microsensor Networks. In Proceedings of the Hawaii International Conference on Systems Sciences, Maui, HI, the U.S.A., 4-7 January 2000; pp. 1-10.

14. Younis, O.; Fahmy, S. HEED: A Hybrid, Energy-Efficient, Distributed Clustering Approach for Ad Hoc

15. Sensor Networks. IEEE Trans. Mob. Comput. 2004, 3, 366-379.

\section{AUTHOR'S PROFILE}

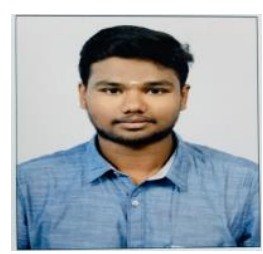

S.Venkatesan, is a Research Scholar in Madurai Kamaraj University, Madurai, Tamil Nadu, India. His main research area includes Network Security, Cryptography and Cloud.

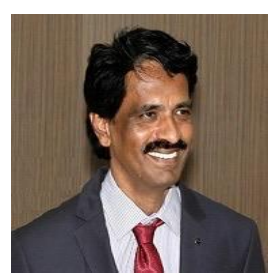

M. Ramakrishnan, is working as the Professor and Head of the Department of Computer Applications, Madurai Kamaraj University, Madurai. His research area includes Network Security, Cloud and Big Data. He has published more than 200 International Journals in various topics.

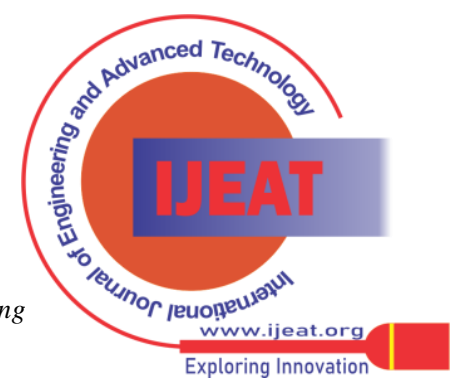

